\title{
Criocoagulación de venas pulmonares como tratamiento complementario de la fibrilación auricular en cirugía valvular
}

\author{
Ricardo Zalaquett S, Erika Pérez A, Bernardita G arayar P, \\ Ismael Vergara S, Sergio Morán V, Pedro Becker R, \\ Manuel J. Irarrázaval LI, Renato 0 choa Ta. \\ Cryoablation of pulmonary veins \\ as complementary treatment \\ of atrial fibrillation in valvular surgery
}

Background: The Cox MAZE III operation for the treatment of atrial fibrillation (AF) is complex and consumes significant operative time. Cryoablation of the pulmonary veins (CPV) is a simpler alternative for patients that require concomitant valvular surgery. Aim: To evaluate CPV in patients with AF submitted to valvular surgery. Patients and Methods: Twenty one patients had simultaneous valvular surgery and $\mathrm{CPV}, 81 \%$ of them had permanent $\mathrm{AF}$ for an average of 5 years. Twenty patients had mitral valve disease. The etiology was rheumatic in 14 . Average left atrial diameter was $60 \mathrm{~mm}$. In 7 patients the mitral valve was replaced, in 5 it was repaired, in 7 both mitral and aortic valve were replaced, in 1 the mitral valve was repaired and the aortic valve was replaced and in 1 only the aortic valve was replaced. A combined transeptal and superior approach was used for all patients. The CPV was performed after the valvular procedure with cryothermy at $-60^{\circ} \mathrm{C}$ for 2 minutes with two $15 \mathrm{~mm}$ cryoprobes applied simultaneously. Results: CPV increased surgical time by 10 to 20 minutes. Operative mortality was $4.8 \%$ (1 patient). One patient developed a pericardial effusion and another a complete heart block that required a permanent pacemaker. All patients improved their functional class. At the end of an average 10.5 months of follow-up, $50 \%$ of patients were in normal sinus rhythm and $25 \%$ persisted in $\mathrm{AF}$. The remaining patients were in some type of regular rhythm. Conclusions: $\mathrm{CPV}$ as a complementary procedure in patients with $\mathrm{AF}$ undergoing valvular surgery had good results to abate AF. It restored normal sinus rhythm in $50 \%$ of the cases, with low morbidity and mortality and little increment in surgical time (Rev Méd Chile 2007; 135: 871-8).

(Key w ords: Atrial fibrillation; Cryosurgery; Heart valves)

Recibido el 14 de julio, 2006. Aceptado el 11 de enero, 2007.

Departamento de Enfermedades Cardiovasculares, Facultad de Medicina, Pontificia Universidad Católica de Chile. Santiago de Chile.

aEnfermero Universitario

Correspondencia a: Dr. Ricardo Zalaquett S. Marcoleta 367,

$6^{\circ}$ piso. Teléfonos 6333030-3543231. Fax 6390108.

E mail: rzalaque@med.puc.cl 
L fibrilación auricular (FA) es una arritmia muy frecuente con una prevalencia en ascenso, lo que se debe, en parte, al envejecimiento progresivo de la población y al consiguiente aumento de las valvulopatías degenerativas ${ }^{1,2}$. En especial, su asociación con la valvulopatía mitral es bien conocida.

Además de los síntomas inherentes a la arritmia completa, la FA permanente se asocia a una duplicación de la mortalidad general y de la mortalidad cardiovascular ${ }^{3-5}$. De aquí el temprano interés en desarrollar procedimientos quirúrgicos que permitiesen «curar» esta amitmia, lo que se concretó con la creación de la operación de maze por James Cox, a comienzos de la década 1990-996-8. Esta operación de Cox, maze, que persigue interrumpir quirúrgicamente los múltiples circuitos de reentrada, base de la FA, tendría éxito en más de $90 \%$ de los casos en la experiencia de $\operatorname{Cox}^{9}$. Sin embargo, es una operación compleja, con morbimortalidad propia y que, efectuada como procedimiento complementario a otra cirugía cardiovascular, incrementa considerablemente los tiempos quinúrgicos.

Por otra parte, actualmente hay consenso que la FA requiere de un sustrato como inflamación o fibrosis auricular y de un elemento desencadenante, como una extrasístole o una alteración de la tensión de la pared auricular, ${ }^{5}$. Si bien el sustrato y el factor desencadenante pueden variar, de acuerdo a la experiencia clínica y a las evidencias experimentales, las venas pulmonares y la aurícula izquierda serían de primordial importancia en la iniciación y mantenimiento de la $\mathrm{FA}^{11,12}$. Así, en la mayoría de los casos, la FA paroxística se originaría a partir de latidos ectópicos originados alrededor de las venas pulmonares y la FA persistente o permanente tendría su origen en la pared posterior de la aurícula izquierda y, también, posiblemente, en las venas pulmonares ${ }^{13-15}$.

Con estas bases, Sueda et al diseñaron para pacientes con FA persistente y valvulopatía mitral, un procedimiento centrado en la aurícula izquierda, que interrumpiría la $\mathrm{FA}$ en $78 \%$ de los casos $^{16,17}$. Este procedimiento comprende la aislación de las venas pulmonares, la resección de la orejuela izquierda y lesiones que conectan hacia el anillo mitral.

A partir de estas observaciones, se desarrollaron una serie de otros procedimientos centrados en la aurícula izquierda y las venas pulmonares, conocidos como «maze parciales», los que utilizan distintas fuentes de energía, como radiofrecuencia, microondas y criotermia ${ }^{16-21}$.

Con estos antecedentes, iniciamos en agosto de 2002 nuestra experiencia en maze parcial izquierdo, como procedimiento complementario para tratar la FA en pacientes valvulares, que requirieron un procedimiento quirúrgico. Nuestra elección, entre los múltiples procedimientos disponibles, fue la criocoagulación de las venas pulmonares (CVP) efectuada a través de un acceso auricular izquierdo y transeptal superior combinado. Los resultados de esta experiencia son el motivo de esta comunicación.

\section{PACIENTES Y MÉTODO}

Pacientes. Se identificaron 21 pacientes sometidos a cirugía valvular en los que se efectuó, concomitantemente, una CVP por FA. Once pacientes $(52,4 \%)$ eran hombres. La edad promedio fue 60 años, con un margen de 36 a 78 años. Los pacientes fueron operados entre agosto de 2002 y julio de 2004 y la indicación de la CVP fue a criterio del cirujano, el que para su decisión tomó en consideración factores intraoperatorios, como la exposición de la aurícula izquierda y la duración del procedimiento valvular. A todos los pacientes se les explicó preoperatoriamente la eventualidad de efectuar este procedimiento complementario.

En 17 pacientes (81\%), la FA era permanente y en $4(19 \%)$ paroxística, de acuerdo a la clasificación del comité conjunto del American College of Cardiology/American Heart Association/European Society of Cardiology 22.

La duración promedio de la FA en los pacientes con FA permanente, fue de 5 años, con un margen de 7 meses a 25 años.

Veinte pacientes (95\%) tenían compromiso de la válvula mitral; en 12 de ellos sólo esta válvula estaba comprometida. El paciente restante tenía una enfermedad valvular aórtica.

La etiología de la valvulopatía mitral fue reumática en 14 casos, mixomatosa en 4 , isquémica en 1 y congénita en otro. En el paciente con enfermedad valvular aórtica, ésta fue degenerativa.

El diámetro ecocardiográfico de la aurícula izquierda fue, en promedio, de $60 \mathrm{~mm}$, con un margen de 50 a $78 \mathrm{~mm}$. 
El $57 \%$ de los pacientes estaba en capacidad funcional (CF) III-IV y 7 (33\%) tenían antecedentes de eventos embólicos. Dos de ellos habían presentado más de un evento. Diez pacientes (48\%) estaban en tratamiento con amiodarona, 6 (29\%) con digoxina y $2(10 \%)$ con ambos. Dieciocho pacientes (86\%) estaban con tratamiento anticoagulante.

En 7 pacientes (33\%) se efectuó un reemplazo mitral, en 5 (24\%) una reparación mitral, en 7 (33\%) un reemplazo mitral y aórtico, en 1, una reparación mitral y reemplazo aórtico y en el paciente restante, un reemplazo valvular aórtico con prótesis biológica (Tabla 1). De los 14 casos con reemplazo mitral, en 13 se utilizó una prótesis mecánica y en 1, una prótesis biológica. La elección del tipo de prótesis dependió de la edad de los pacientes, prefiriéndose las prótesis mecánica en los pacientes menores de 70 años. La Figura 1 resume el tipo de prótesis utilizada.

En 8 pacientes (38\%) se efectuó, además, una anuloplastia tricuspídea y en 3 (14\%) una revascularización miocárdica. Igualmente, $14 \%$ de los casos correspondieron a reoperaciones.

Procedimiento quirúrgico. Todos los pacientes fueron operados con circulación extracorpórea con hipotermia de $30^{\circ} \mathrm{C}$, para lo cual se canuló la aorta y ambas cavas con cánulas de pequeño calibre. El drenaje venoso se efectuó con presión negativa controlada. La protección miocárdica se efectuó con solución cardioplégica cristaloide infundida a $4^{\circ} \mathrm{C}$ por vía anterógrada y retrógrada.

Se utilizó un acceso auricular izquierdo transeptal y superior combinado, en todos los $\operatorname{casos}^{23}$. Para esto, se efectúa una amplia auriculotomía derecha paralela, y a $2 \mathrm{~cm}$, al surco aurículoventricular, desde la proximidad de la cava inferior hasta la unión de la porción superior y medial de la aurícula derecha. Una vez canulado el seno coronario, se incide el septum interauricular, desde el limbo inferior de la fosa ovalis, hasta unirse

\section{Tabla 1. Procedimiento valvular efectuado en 21 pacientes con criocoagulación de venas pulmonares, como tratamiento complementario de la fibrilación auricular}

\begin{tabular}{|lcc|}
\hline & № pacientes & $\%$ \\
\hline Reemplazo mitral & 7 & 33 \\
Reemplazo mitroaórtico & 7 & 33 \\
Reparación mitral & 5 & 24 \\
Reparación mitral- & & \\
$\quad$ reemplazo aórtico & 1 & 5 \\
Reemplazo aórtico & 1 & 5 \\
\hline
\end{tabular}

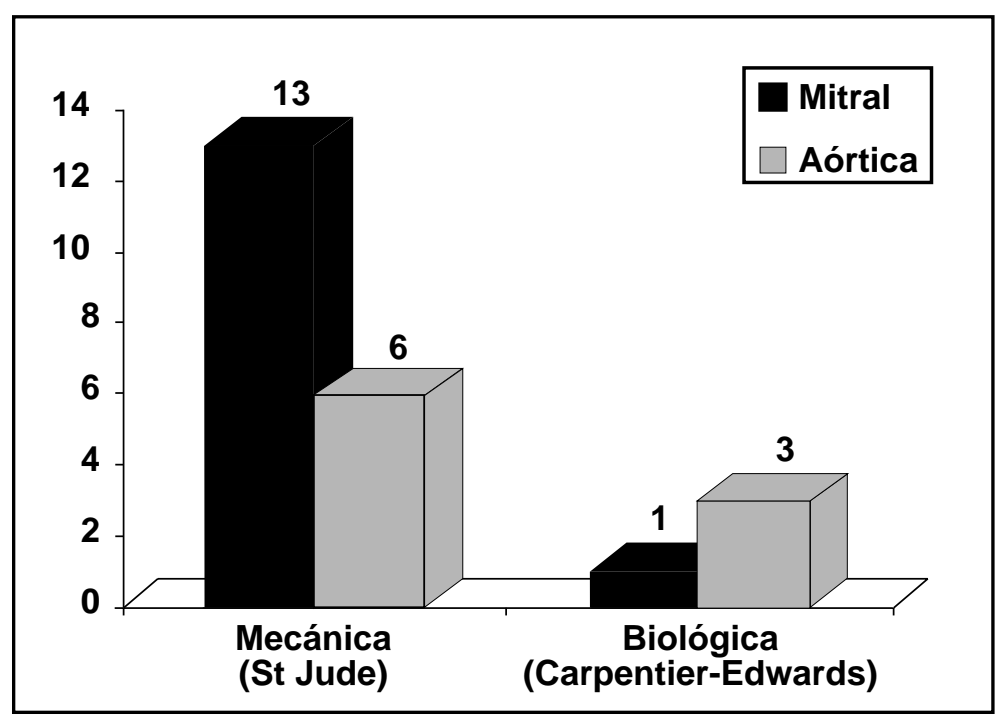

Figura 1. Tipo de prótesis valvular utilizada según posición mitral o aórtica. 
a la incisión auricular derecha previa. Se continúa luego la incisión por el techo de la aurícula izquierda, bajo la aorta ascendente, hasta la base de la orejuela izquierda. Se crean así, extensas incisiones quirúrgicas en el septum interauricular y techo de la aurícula izquierda.

Una vez efectuado el procedimiento mitral, se procede a la criocoagulación del piso de la aurícula izquierda, alrededor de los ostia de las venas pulmonares, utilizando una máquina Frigitronic ${ }^{\circledR}$ de 2 salidas, especialmente diseñada para cirugía cardíaca, en la que la base del enfriamiento es el óxido nitroso. En la mayoría de los casos, se utilizan 2 sondas de $15 \mathrm{~mm}$ para efectuar simultáneamente 2 lesiones hipotérmicas transmurales, criocoagulando a $-60^{\circ} \mathrm{C}$ por $2 \mathrm{~min}$. La descongelación es efectuada por la máquina y complementada con la irrigación de solución fisiológica.

El procedimiento se inicia alrededor de las venas pulmonares izquierdas y se avanza progresivamente hacia las venas pulmonares derechas, para luego efectuar una o más lesiones hacia el anillo mitral, hasta llegar a éste y otras, hasta la base de la orejuela izquierda (Figura 2).

En los 14 pacientes en que se cerró la orejuela izquierda, que corresponden a los últimos de la serie, esto se efectuó a continuación de la CVP con una sutura continua de Prolene ${ }^{\circledR}$ 3-0.

La criocoagulación del istmo cavo-tricuspídeo se efectuó luego del cierre de la auriculotomía izquierda, criocoagulando a $-60^{\circ} \mathrm{C}$ por 2 min con ambas sondas de $15 \mathrm{~mm}$ simultáneamente, por una sola vez, entre el anillo tricuspídeo y la llegada de la cava inferior.

Tratamiento antiarrítmico. El tratamiento antiarrítmico postoperatorio fue efectuado libremente por el cardiólogo tratante, sin un protocolo antiarrítmico definido previamente. Igualmente, la decisión de cardiovertir eléctricamente a los pacientes con recurrencia de la FA, fue también de éste.

Método. Los pacientes fueron identificados del registro de criocoagulación del Servicio de Cirugía Cardiovascular de nuestra Institución, el que incorpora a todos aquellos pacientes en los que la máquina Frigitronic es utilizada.

La información se obtuvo de la revisión de las fichas clínicas y protocolos operatorios.

A todos los pacientes se les efectuó un electrocardiograma y un ecocardiograma transtorácico previo al alta hospitalaria.

Seguimiento. Todos los pacientes fueron contactados en agosto de 2004, solicitándoseles responder un cuestionario clínico dirigido a determinar $\mathrm{CF}$, palpitaciones o cualquier otra manifestación que estos consideraran sugerente de arritmia. Igualmente, a todos los pacientes se les efectuó en ese momento un electrocardiograma. Sin embargo, no a todos los pacientes se les efectuó un ecocardiograma alejado, por lo que no fue posible objetivar la existencia de contracción auricular efectiva a través de análisis de flujo.
Figura 2. Esquema de maze parcial izquierdo efectuado. Las venas pulmonares son aisladas en un «cuadrado»con lesiones de criocoagulación, del que se desprenden lesiones que conectan hacia el anillo mitral y la orejuela izquierda. El ostium de ésta es suturado.

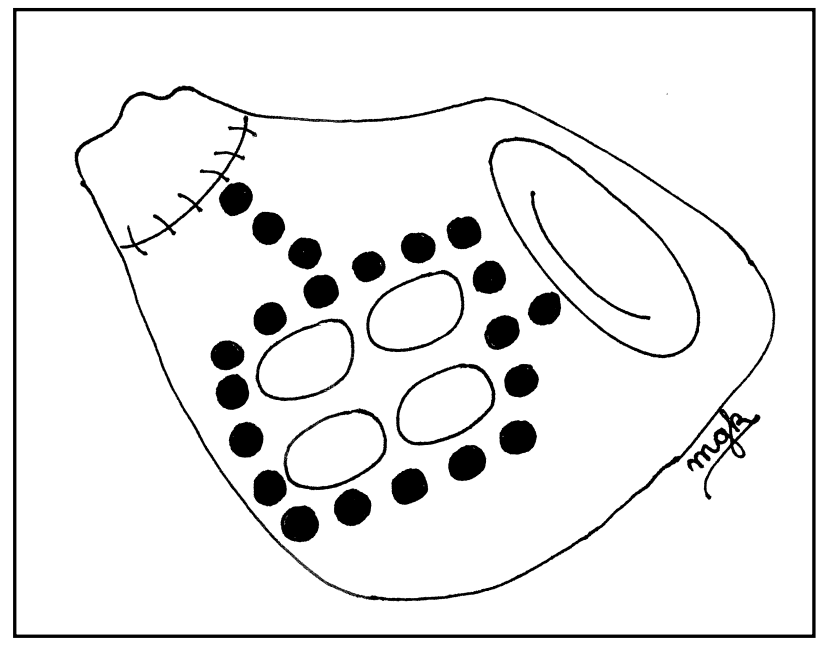




\section{RESULTADOS}

El procedimiento se efectuó sin incidentes en todos los pacientes en que fue programado. Se efectuaron, en promedio, 10 criocoagulaciones auriculares izquierdas por paciente. En la mayoría de los casos, éstas se efectuaron de a 2 simultáneamente, utilizando ambas sondas de criocoagulación.

Así, se estimó que la CVP significó una adición entre 10 y 20 min al tiempo operatorio, para los 20 pacientes primariamente mitrálicos.

Por otra parte, la criocoagulación del istmo cavo-tricuspídeo, la que se efectuó en 5 pacientes con antecedentes de flutter auricular, significó una adición al tiempo quirúrgico de 2 min.

Mortalidad operatoria. Un paciente falleció por insuficiencia cardíaca, por lo que la mortalidad operatoria fue 4,8\%. Éste fue un hombre de 78 años, con grave disfunción ventricular izquierda y FA de difícil control, en quien se efectuó un reemplazo mitral y aórtico y una anuloplastia tricuspídea, así como una revascularización miocárdica con 2 puentes de vena safena, además de la CVP.

Morbilidad operatoria. Un paciente presentó un derrame pericárdico que requirió de drenaje. Otro paciente presentó un bloqueo aurículo-ventricular completo, por lo que previo a su alta se implantó un marcapaso bicameral. Ningún paciente fue reintervenido por hemorragia posoperatoria.

Ritmo cardíaco prealta hospitalaria. Seis pacientes (30\%) se encontraban en FA al momento de su alta. Catorce pacientes (70\%) presentaban un ritmo regular, en 12 de los cuales (60\%), éste era sinusal.

Seguimiento. El seguimiento se completó en 100\% de los casos, con un promedio de 10,5 meses y un margen de 1 a 22 meses.

Mortalidad alejada. Todos los pacientes estaban vivos al término del seguimiento.

Morbilidad alejada. No hubo morbilidad en el seguimiento. Especialmente, ningún paciente presentó un evento tromboembólico.

Capacidad funcional. Al cierre del seguimiento los 20 pacientes sobrevivientes se encontraban en CF I-II.
Ritmo cardíaco al término del seguimiento. En 4 pacientes se efectuó una cardioversión eléctrica luego del alta hospitalaria. Ésta fue exitosa en todos ellos, los que pasaron a ritmo sinusal.

$\mathrm{Al}$ término del seguimiento, 5 pacientes (25\%) se encontraban en FA y 10 (50\%) estaban en ritmo sinusal, 4 de los cuales correspondieron a los pacientes con FA paroxística preoperatoria. Los pacientes restantes estaban en algún tipo de ritmo regular: tres (15\%) presentaban un ritmo auricular, $1(5 \%)$ ritmo nodal y otro (5\%) ritmo de marcapaso. Así, $75 \%$ de los pacientes estaban libres de FA (Tabla 2).

Tratamiento antiarrítmico. El 90\% de los pacientes estaba recibiendo algún medicamento antiarrítmico al término del seguimiento. Siete pacientes (35\%) recibían amiodarona, 3 (15\%) betabloqueadores, 1 (5\%) digoxina y $6(30 \%)$ algún otro medicamento antiarrítmico.

Tratamiento anticoagulante. Quince pacientes (75\%) estaban con tratamiento anticoagulante al término del seguimiento. En sólo 2 casos la indicación primaria fue FA recurrente. Los otros 13 eran portadores de una prótesis mitral mecánica.

\section{Discusión}

En relación al tratamiento quirúrgico de la FA es necesario distinguir 2 situaciones: una es aquella FA recurrente, no asociada a valvulopatía mitral y otra, aquella FA secundaria a ésta, con repercu-

\section{Tabla 2. Ritmo cardíaco al término del seguimiento para 20 pacientes sobrevivientes con criocoagulación de venas pulmonares, como tratamiento complementario de la fibrilación auricular en cirugía valvular}

\begin{tabular}{|lrr|}
\hline & No pacientes & $\%$ \\
\hline Sinusal & 10 & 50 \\
Auricular & 3 & 15 \\
Nodal & 1 & 5 \\
Marcapaso DDD & 1 & 5 \\
Fibrilación auricular & 5 & 25 \\
\hline
\end{tabular}


sión en la aurícula izquierda. Ambas situaciones son susceptibles de tratamientos quirúrgicos y, si bien el éxito de cualquier procedimiento es mayor en la primera situación, éstos se utilizan actualmente con mayor frecuencia como un procedimiento asociado a una cirugía cardíaca, en particular mitral $21,25,26$. Igualmente, la operación de Cox-maze tiene su plena indicación como tratamiento de la FA «solitaria». Sin embargo, ésta continúa siendo una operación compleja, con morbimortalidad propia y que asociada a otro procedimiento cardiovascular prolonga excesivamente los tiempos operatorios. De aquí que como procedimiento complementario, los maze parciales izquierdos se hayan impuesto por sobre la operación de Cox-maze, a pesar de que su éxito alejado sería menor, del orden de 70\%16-20,24.

¿Por qué la criocoagulación? De todas las distintas fuentes energéticas disponibles para efectuar un maze parcial, nuestra elección fue por la criotermia, si bien la radiofrecuencia sería más popular, por varias razones. La criocoagulación es un procedimiento bien establecido en cirugía de arritmia y es un componente esencial de la operación de Coxmaze. Aplicada a $-60^{\circ} \mathrm{C}$ por $2 \mathrm{~min}$, produce lesiones transmurales, demostradas experimentalmente, lo que es un requisito para cualquier procedimiento, a la vez que preserva la arquitectura tisular y no altera la regularidad de la superficie endocárdica ${ }^{6-8}$.

Igualmente, la criocoagulación fue la fuente energética elegida por Sueda en su trabajo pionero con maze parcial izquierdo ${ }^{16,17}$. Pero, independientemente de lo anterior, una razón importante fue la disponibilidad de una máquina de criocoagulación especialmente diseñada para cirugía de arritmias, la que además no requiere de elementos desechables de elevado costo. Las caractenísticas de ésta, permite efectuar 2 lesiones hipotérmicas simultáneamente, lo que facilita un procedimiento rápido y expedito.

En nuestra experiencia, la rigidez de las sondas de criocoagulación no ha sido un inconveniente, como se ha reportado, probablemente por el acceso auricular izquierdo transeptal y superior utilizado, el que da amplia accesibilidad a toda la aurícula izquierda ${ }^{23,26}$.

Además, este acceso, implica efectuar extensas incisiones quirúrgicas en la aurícula derecha, aislando parcialmente la orejuela derecha y la llegada de la cava superior, en el septum interauricular, seccionando los limbos inferior y superior de la fosa ovalis y en el techo de la aurícula izquierda, lo que de alguna manera podría también participar en la interrupción de la FA.

En la actualidad, en todos los pacientes, además del cierre de la orejuela izquierda, efectuamos la criocoagulación del istmo cavo-tricuspídeo, puesto que, aunque no ha sido nuestra experiencia, existiría un mayor riesgo de presentar flutter auricular en los maze izquierdos, el cual se originaría en la aurícula derecha ${ }^{27}$.

¿Por qué efectuar un maze izquierdo en recambios mitrales con prótesis mecánica? Si bien la FA se asocia principalmente con el riesgo de tromboembolismo y la necesidad de tratamiento anticoagulante, que será de todas formas necesario en pacientes con prótesis mecánica, también tiene un efecto adverso en el gasto cardíaco, al perderse la contribución de la aurícula izquierda al volumen expulsivo del ventrículo izquierdo. Por otra parte, en muchos pacientes, la percepción de palpitaciones irregulares resulta desagradable, lo que puede ser de difícil control farmacológico, siendo necesario, en algunos casos, recurir a la ablación del nódulo aurículoventricular. De aquí la importancia de recuperar un ritmo sinusal, en especial en pacientes con disfunción ventricular, o en su defecto, regularidad en los latidos cardíacos, en particular en pacientes portadores de prótesis mecánica en quienes, además de percibir la arritmia, el cierre y apertura de la prótesis resulta frecuentemente audible.

¿Fue el procedimiento exitoso en cuanto a suprimir la FA? Evaluar y comparar los resultados de los distintos procedimientos para la ablación de la FA es difícil, puesto que implica la existencia de definiciones universalmente aceptadas, en cuanto a seguimiento de los pacientes, de la arritmia, del análisis de los resultados y de la forma en que éstos se reportan, lo que no es del todo claro que así sea en la actualidad ${ }^{26}$. Igualmente, es distinto evaluar los resultados en cuanto a FA primaria, no secundaria a valvulopatía mitral, que cuando ésta es precisamente por esta situación. Además, la etiología de la valvulopatía mitral, así como el tamaño de la aurícula izquierda, son muy determinantes en cuanto al éxito de un procedimiento para suprimir la $\mathrm{FA}^{24,28}$.

Nuestros pacientes fueron en su mayoría reumáticos con enfermedad mitral de larga data e importante repercusión en la aurícula izquierda, en que el diámetro promedio de ésta fue de $60 \mathrm{~mm}$ y 
el máximo $78 \mathrm{~mm}$, lo que significa que en muchos casos el miocardio auricular ya había sido sustituido por tejido fibroso. Además, la FA tenía una duración promedio de 5 años.

Como quiera que sea, $75 \%$ de los pacientes al cierre del seguimiento, es decir, en promedio a los 10,5 meses de la operación, se encontraba libre de FA y $50 \%$ en ritmo sinusal. Por otra parte, si bien la mayoría de éstos se encontraban en tratamiento antiarrítmico, en sólo 4 se había efectuado una cardioversión eléctrica luego del alta hospitalaria, la que fue exitosa en todos, puesto que éste fue un estudio estrictamente observacional, a diferencia de la mayoría de los trabajos publicados en los que la cardioversión es efectuada en prácticamente todos los pacientes que persisten en FA.

Por último, sólo 1 paciente (5\%) presentó morbilidad que podría ser atribuida al procedimiento, consistente en un bloqueo aurículo-ventricular completo que requirió del implante de marcapaso. En la experiencia de Cox con maze III, el 15\% de los pacientes requirió de un marcapaso ${ }^{9}$. No hubo complicaciones temibles, como las presentadas con la radiofrecuencia, como es la fístula aurículoesofágica, o con las microondas, como es la lesión del tronco de la arteria coronaria izquierda ${ }^{29-31}$.

Así, si bien el procedimiento efectuado por nosotros tendría resultados algo menores a los

\section{REFERENCIAS}

1. Sra J, Dhala A, Blanck Z, Deshpande S, Cooley R, AкHTAR M. Atrial fibrillation: epidemiology, mechanisms, and management. Curr Probl Cardiol 2000; 25: 405-24.

2. Feinberg W, Blackshear J, Laupacis A, Kronmal R, HART R. Prevalence, age distribution, and gender of patients with atrial fibrillation. Analysis and implications. Arch Intern Med 1995; 155: 469-73.

3. Kannel W, Abbott $R$, Savage D, McNamara P. Epidemiologic features of chronic atrial fibrillation: the Framingham study. N Engl J Med 1982; 306: 1018-22.

4. Benjamin E, Wolf P, D'agostino R, Silbershatz H, KanNeL W, LeVY D. Impact of atrial fibrillation on the risk of death: the Framingham Heart Study. Circulation 1998; 98: 946-52.

5. FALK R. Atrial fibrillation. New Engl J Med 2001; 344: 1067-78. comunicados en la literatura en cuanto a restituir un ritmo sinusal, sí puede ser considerado exitoso en cuanto a suprimir la FA en la mayoría de los casos, con baja morbimortalidad, incremento limitado del tiempo operatorio y bajo costo.

Limitaciones del estudio. Este fue un estudio estrictamente observacional y descriptivo de un procedimiento complementario a otra cirugía, por lo que tiene todas las limitaciones propias de este tipo de estudios, pero representa bien las características de los pacientes valvulares en Chile, con fuerte predominio reumático, importante crecimiento auricular izquierdo y fibrilación auricular de larga data. Sin embargo, los resultados, si bien iniciales, son alentadores, por lo que hemos continuado su uso y eventualmente comunicaremos resultados en un mayor número de pacientes con un seguimiento más largo.

Conclusión. La CVP a través de un acceso auricular izquierdo transeptal y superior combinado como procedimiento complementario a una cirugía cardíaca, en especial mitral, en pacientes portadores de FA recurrente, tendría buenos resultados en cuanto a abolir la FA, con baja morbimortalidad intrínseca y escaso incremento en los tiempos quirúrgicos y en los costos operatorios.

6. Cox J, Schuessier R, D'agostino H Jr, Stone C, Chang B, CAIN M ET AL. The surgical treatment of atrial fibrillation. III. Development of a definitive surgical procedure. J Thorac Cadiovasc Surg 1991; 101: 569-83.

7. Cox J, Boineau J, Schuessier R, Jaquiss R, Lappas D. Modification of the maze procedure for atrial flutter and atrial fibrillation. I. Rationale and surgical results. J Thorac Cardiovasc Surg 1995; 110: 473-84.

8. Cox J, Jaquiss R, Schuessier R, Boineau J. Modification of the maze procedure for atrial flutter and atrial fibrillation. II. Surgical technique of the maze III procedure. J Thoracic Cardiovasc Surg 1995; 110: 485-95.

9. Cox J, Ad N, Palazzo T, Fitzpatrick S, Suyderhoud J, Degroot $K$ et al. Current status of the maze procedure for the treatment of atrial fibrillation. Semin Thorac Cardiovasc Surg 2000; 12: 15-9.

10. NatTel S. New ideas about atrial fibrillation 50 years on. Nature 2002; 415: 219-26. 
11. Epstein A, Kay G. Finding our way through the maze. J Cardiovasc Electrophysiol 1999; 10: 1575-7.

12. RoITHinger F, Steiner P, Goseki Y, Sparks P, Lesh M. Electrophysiologic effects of selective right versus left atrial linear lesion in a canine model of chronic atrial fibrillation. J Cardiovasc Electrophysiol 1999; 10: 1564-74.

13. Haissaguerre M, Jais $P$, Shah DC, Atsushi $T$, MélĖze H, GILES Q ET AL. Spontaneous initiation of atrial fibrillation by ectopic beats originating in the pulmonary veins. N Engl J Med 1998; 339: 659-66.

14. Nathan $H$, Euakim M. The junction between the left atrium and the pulmonary veins. An anatomic study of human hearts. Circulation 1966; 34: 412-22.

15. Harada A, Sasaki K, Fukushima T, Ikeshita M, Asano T, YAMAUCHI S ET AL. Atrial activation during chronic atrial fibrillation in patients with isolated mitral valve disease. Ann Thorac Surg 1996; 61: 104-12.

16. Sueda T, Nagata H, Orihashi K, Morita S, Okada K, Sueshiro M ET aL. Efficacy of a simple left atrial procedure for chronic atrial fibrillation in mitral valve operations. Ann Thorac Surg 1997; 63: 1070-5.

17. Sueda T, Nagata $H$, Shikata $H$, Orihashi $K$, Morita $S$, SuESHIRO M ET AL. Simple left atrial procedure for chronic atrial fibrillation associated with mitral valve disease. Ann Thorac Surg 1996; 62: 1796800.

18. Wimams M, Stewart J, Bown S, Freeman S, Anderson J, Argenziano M et aL. Surgical treatment of atrial fibrillation using radiofrequency energy. Ann Thorac Surg 2001; 71: 1939-44.

19. Sie H, Beukema W, Misier A, Elvan A, Ennema J, HaAlebos M ET aL. Radiofrequency modified maze in patients with atrial fibrillation undergoing concomitant cardiac surgery. J Thorac Cardiovasc Surg 2001; 122: 249-56.

20. Sitzer S, Richter P, Knaut M, Schuler S. Treatment of atrial fibrillation in open heart surgery-the potential role of microwave energy. Thorac Cardiovasc Surg 1999; 47: 374-8.

21. Cox J. Intraoperative options for treating atrial fibrillation associated with mitral valve disease. J Thorac Cardiovasc Surg 2001; 122: 212-5.

22. Fuster V, Ryden L, Asinger R, Cannom D, Crijns $H$, FRYE RL ET AL. ACC/AHA/ESC guidelines for the management of patients with atrial fibrilation. A report of the American College of Cardiology/ American Heart Association Task Force on Practice Guidelines and the European Society of Cardiology Committee for Practice Guidelines and Policy Conferences (Committee to develop guidelines for the management of patients with atrial fibrillation) developed in collaboration with the North American Society of Pacing and Electrophysiology. Eur Heart J 2001; 22: 1852-923.

23. Zalaquett R, Morán S, Irarrázaval M, Maturana G, NAVARRO M, Braun S et al. Acceso auricular izquierdo transeptal y superior combinado. Rev Chil Cirugía 1993; 45: 225-8.

24. Izumoto $\mathrm{H}$, Kawazoe $\mathrm{K}$, Kitahara $\mathrm{H}$, Kamata J. Operative results after the Cox/maze procedure combined with a mitral valve operation. Ann Thorac Surg 1998; 66: 800-4.

25. Patwardhan A, Dave $H$, Tamhane A, Pandit S, Dalvi B, Golam K eT AL. Intraopeative radiofrequency microbipolar coagulation to replace incisions of maze III procedure for correcting atrial fibrillation in patients with rheumatic valvular disease. Eur J Cardiothorac Surg 1997; 12: 627-33.

26. Giwnov A, Biackstone E, McCarthy P. Atrial fibrillation: Current surgical options and their assessment. Ann Thorac Surg 2002; 74: 2210-17.

27. Usui A, Inden Y, MizUtani S, TaKagi Y, AKITA T, Ueda Y. Repetitive atrial flutter as a complication of the left-sided simple maze procedure. Ann Thorac Surg 2002; 73: 1457-9.

28. Kamata J, Kawazoe K, Izumoto H, Kitahara H, Shina Y, Sato Y ET al. Predictors of sinus rhythm restoration after Cox maze procedure concomitant with other cardiac operations. Ann Thorac Surg 1997; 64: 394-8.

29. Giwnov A, Pettersson G, Rice T. Esophageal injury during radiofrequency ablation for atrial fibrillation. J Thorac Cardiovasc Surg 2001; 122: 123940.

30. Mohr F, Fabricius A, Falk V, Autschbach R, Doll N, VON OPPELL U ET AL. Curative treatment of atrial fibrillation with intraoperative radiofrequency ablation: short-term and midterm results. J Thorac Cardiovasc Surg 2002; 123: 919-27.

31. Manasse E, Medici D, Ghisew S, Ornaghi D, GашоттI R. Left main coronary arterial lesion after microwave epicardial ablation. Ann Thorac Surg 2003; 76: 276-7. 